\title{
Facilitating Endotracheal Intubation in Difficult Cases Using an External Magnet
}

Mahin Seyedhejazi ${ }^{1^{*}}$, Masood Banaii ${ }^{2}$, Nasrin Taghizadieh ${ }^{3}$, Mahni Mokaber $^{4}$

1. Department of Anesthesiology, Tabriz University of Medical Sciences, Tabriz, Iran

2. Department of Biomedical Engineering, School of Electrical and Computer Engineering, College of Engineering, University of Tehran

3. Tabriz University of Medical Sciences, Tabriz, Iran

4. Medical Education Research Center, Tabriz University of Medical Sciences, Tabriz, Iran

\section{Introduction}

Difficult airway is conventionally defined as a medical scenario in which a trained examiner faces difficulty in either facemask ventilation or tracheal intubation (1). Unlike difficult intubation, the incidence of difficult mask ventilation in adults is considerable $(2,3)$. Anesthesiologists and those who practice intubation should be familiar with the management of airway and be able to recognize and identify potentially difficult airways including congenital craniofacial deformities with micrognathia (e.g. Pier Robin, Treacher Collins, Goldenhar's, and Crouzon's syndromes) and metabolic diseases causing the deposition of accumulated by-products (e.g., Hurler's, Morquio's, and Beckwith-Wiedemann syndromes). Cormack and Lehane grades 3 and 4 at laryngoscopy are an indication for advanced techniques for intubation. Laryngeal mask airway (LMA) and fiberscope with a directable tip are useful and important modalities in handling difficult airway and intubation (5).

Even normal pediatric airway could become critical due to the anatomical and physiological differences between pediatric and adult airway; this particularly becomes a concern in infants, i.e. children younger than one year old. This hazard is augmented in the presence of congenital or acquired difficulties affecting airway. Consequently, proper preoperative assessment is considered as the cornerstone of pediatric difficult airway management. Every anesthetic plan should be tailored according to patients considering the scenario and also the expertise of the practitioner. Opting for spontaneous respiration maintenance and intervening in a step-wise manner are strongly suggested (6).

Multiple airway devices have been and are developed that all of which can be placed under direct vision or blindly; most of these devices consistently both provide and maintain

\section{Corresponding author:}

Mahin Seyedhejazi, MD

Associate Professor of Pediatric Anesthesia, Tabriz University of Medical, Tabriz, Iran Sciences, Children Hospital, Sheshkalan St

E-mail: seidhejazie@yahoo.com Fax: 00984135262279 Tel: 00989141150981

Receive date: 2014-09-25| Accept date: 2014-10-10| Publish date: 2014-10-30

DOI: 10.7575/aiac.abcmed.15.03.02.11 
safe oxygenation and ventilation. Furthermore, a wide range of ancillary devices have also been introduced to be of assistance in the management of difficult airway; most of them are available in varied sizes suitable for use in children and incorporate a variety of different types of endotracheal tubes, supraglottic devices, fiber-optic, video, optical, and mechanical technologies. Some of these devices (e.g. video laryngoscope) are expensive and not suitable for developing countries with limited financial reserve; some others need high levels of experiences or learning courses which is not possible for anesthesiologist that are not working in university hospitals or are less experienced. Some of these devices cannot be used in small children or neonates. Therefore, we were encouraged to look for a device that is easy to use and carry, inexpensive, small in size, and available in all sizes for children, and also does not need special training.

\section{Methods}

For every anesthesiologist, especially for less experienced ones, it happens that in spite of visualizing vocal cords in direct laryngoscopy, the endotracheal tube (ETT) softly slides caudally and posteriorly even with stylet (a wire-like device that is used in difficult airway management for direction of ETT) and we cannot redirect tube and intubate the patient. Therefore, if we could make a device to prevent ETT from sliding caudally, we might prevent many cases of failed intubation. Our hypothesis is that if we use a small magnet in the tip of stylet (Fig 1) or on the tip of ETT (Fig 2) while a part of the magnet lying over the neck (Fig 3), due to the power of magnetic field around the tip of ETT, it would slide anteriorly and consequently the tip of tube would be attracted anteriorly making the intubation easier. It seems that this device might be of more educational use where numerous intubation attempts are predicted due to caudal sliding of ETT. This device can be easily used without special training in patients of all sizes. In addition, it will be inexpensive and can be easily carried.

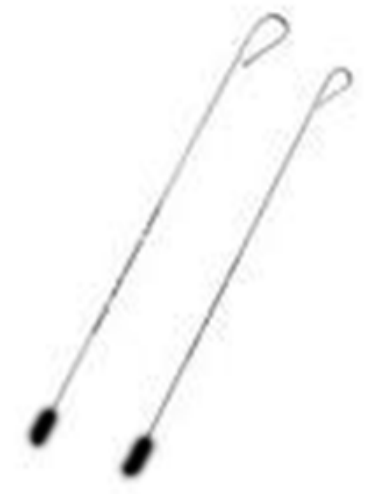

Figure 1: stylet

\section{Discussion}

Every anesthesiologist confronting the difficult pediatric airway is presented with a unique set of challenges. Adult difficult airway

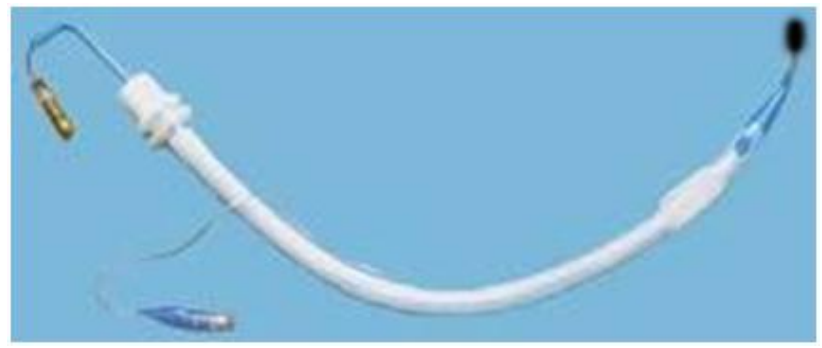

Figure 2: ETT with stylet (magnet on the tip of ETT or on the tip of stylet)

management techniques, such as awake or invasive approaches often cannot be applied to children because of inadequate cooperation. Consequently, awake intubation in pediatrics is uncommon; most intubations are performed under general anesthesia or deep sedation. From a physiologic perspective, children can tolerate apnea period in shorter lengths significantly compared to the adults due to higher rates of oxygen consumption (7). Children are also small in size with limited neck tissue and mass; therefore, the recommended device seems to be practical mostly in children (8). While difficult airway is dreaded as one of 
the leading factors contributing to anesthesiarelated deaths, remarkable advances been made in the field in the last few decades are encouraging (9).

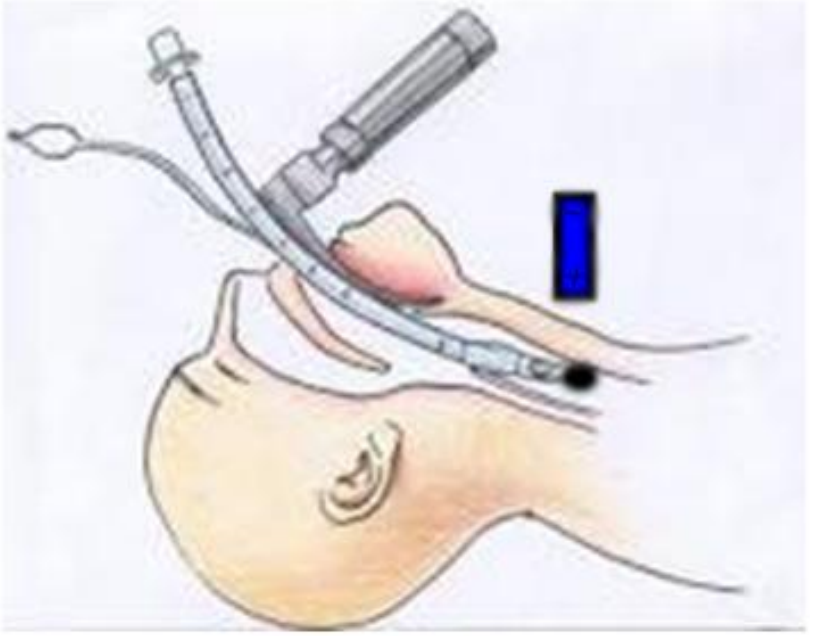

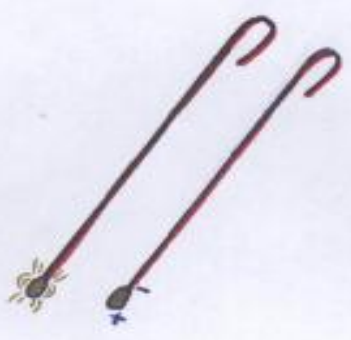
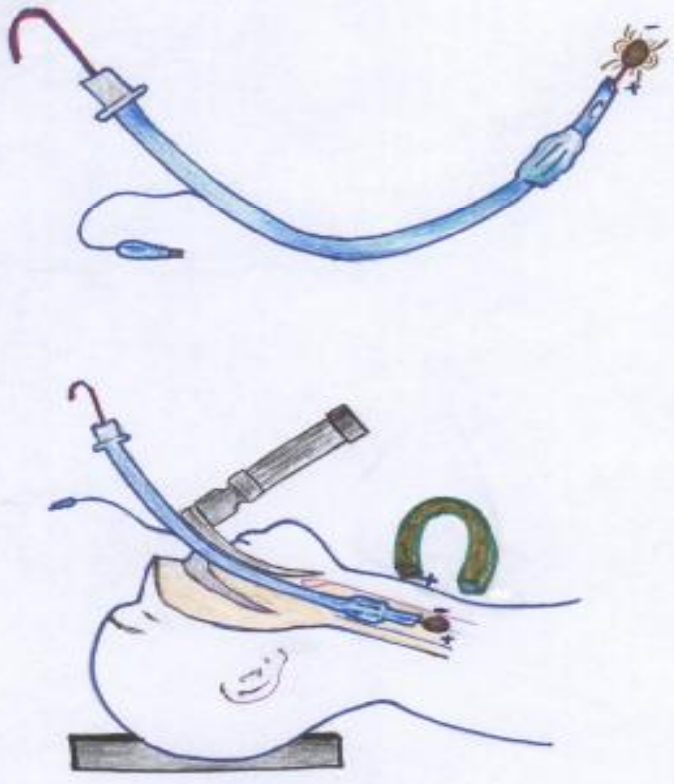

The question is if advanced airway management skills are enough for every case. Prior to learning how to utilize more complicated techniques such as fibreoptic bronchoscopy, gaining experience in common fundamental airway management skills seems inevitable (10).

Difficult airway algorithm for the unexpected difficult airway in pediatric patients includes administration of varied devices such as different types of laryngoscope blades, ETT of various sizes and curves, anesthesia equipment, Bullard laryngoscopes, lighted stylets, retrograde intubation, fiberoptic bronchoscope, rigid bronchoscope, equipment for tracheostomy, etc. 
The recommended device is easy to learn and requires minimal training and practice. It seems that its usage would be less timeconsuming and associated with fewer traumas compared with multiple attempts at direct laryngoscopy, fiberoptic, or blind tracheal intubation in children. We believe that this device could be of valuable assistance in the Figure 3: second part of magnet on the neck management of the anticipated or unanticipated adult and especially pediatric difficult airway. It should be emphasized that there is not any sole ideal device suited for all of difficult intubation cases; however, we assume that this device could have a place among difficult air way devices and even in simple airway management Kits.

\section{References}

1. American Society of Anesthesiologists Task Force on Difficult Airway Management. Practice Guidelines for Management of the Difficult Airway: An Updated Report by the American Society of Anesthesiologists. Anesthesiology 2003 98: 1269-1277.

2. Kheterpal et al. Incidence and predictors of difficult and impossible mask ventilation. Anesthesiology 2006; 105:885-913.

3. Langeron et al. Prediction of difficult mask ventilation. Anesthesiology 2000 92:1229 -36.

4. Tait AR et al. Incidence and risk factors for perioperative adverse respiratory events in children who are obese. Anesthesiology 2008 108:375-80.

5. KinouchiK.Department of Anesthesia \& Intensive Care, Osaka Medical Center and Research Institute for Maternal \& Child Health, [Management of difficult pediatric airway]. Izumi 594-1101,Masui. 2006 Jan;55(1):2432.

6. DrYoshaPrasad,SpRAnaesthesia.Lister Hospital, Stevenage, UK THE DIFFICULT PAEDIATRIC AIRWAY ANAESTHESIA TUTORIAL OF THE WEEK 250 13TH FEBRUARY 2012.

7. Fiadjoe J, Stricker P.Pediatric difficult airway management: current devices and techniques.Department of Anesthesiology and Critical Care Medicine, Children's Hospital of Philadelphia, University of Pennsylvania, Philadelphia, PA 19104, USA. fiadjoej@email.chop.edu,AnesthesiolClin. 2009 Jun;27(2):185-95.

8. S Ramesh and R Jayanthi.Supraglottic airway devices in children. Indian J Anaesth. 2011 Sep-Oct; 55(5): 476482.

9. Pediatric Anesthesia.Volume 14, Issue 1, pages 28-37, January 2004.

10. EtsuroK.Motoyama ,PeterJ.Davis.SMITH'S Anesthesia for Infants and children.2006.350-351. 